\title{
Familial Affective Responsiveness Mediates the Relations Between Parental Perfectionistic Self- Presentation and Adolescent Psychological Maladjustment
}

Chang Chen ( $\square$ chang.chen@psych.ubc.ca )

The University of British Columbia

\section{Agostino Brugnera}

University of Bergamo: Universita degli Studi di Bergamo

Paul L. Hewitt

The University of British Columbia

Carmen F. Caelian

The University of British Columbia

Gordon L. Flett

York University

\section{Research Article}

Keywords: parental perfectionism, adolescent depression, suicidal ideation, alcohol-related problems, affective responsiveness, family functioning

Posted Date: February 3rd, 2022

DOI: https://doi.org/10.21203/rs.3.rs-1317918/v1

License: (c) (i) This work is licensed under a Creative Commons Attribution 4.0 International License.

Read Full License 


\section{Abstract}

Parental perfectionism (e.g., perfectionistic concerns) has been strongly implicated in adolescent psychological maladjustment such as depression and anxiety. However, little is known about the links between parental perfectionistic self-presentation (i.e., the interpersonal expressions of perfectionism) and adolescent psychological outcomes. According to the Perfectionism Social Disconnection Model (Hewitt et al., 2017), people with elevated perfectionistic self-presentation engage in social behaviors characterized by a lack of emotional sensitivity or responsiveness, which then increases one's risk of social disconnection and psychological maladjustment. This theory has seldom been tested in the context of parent-adolescent and family relationships. Therefore, this cross-sectional study aimed to explore the mediational role of familial affective responsiveness in the associations between parental perfectionistic self-presentation and adolescent psychological outcomes. Participants were 58 dyads of clinically depressed adolescents $\left(M_{\mathrm{age}}=15.52 ; 74.5 \%\right.$ female $)$ and their primary caregivers $\left(M_{\mathrm{age}}=46.08\right.$; 51 mothers). Path analysis indicated that the overall model fit the data well. Congruent with the Perfectionism Social Disconnection Model, analyses showed that adolescent but not parent-reported family dysfunction in affective responsiveness mediated the relations between parental perfectionistic self-presentation and adolescent depressive symptoms, suicidal ideation, and alcohol-related problems, even after controlling for parental depression. Taken together, our findings provide new information for the role of parental perfectionistic self-presentation in adolescent psychological maladjustment and highlight parental perfectionistic self-presentation as a potential target for therapeutic intervention in adolescent depression.

\section{Full Text}

Depression is a common and debilitating mental health problem among youth worldwide (Patel et al., 2007). In the United States, it is estimated that one in four adolescents will experience a depressive disorder by the age of 18 (Merikangas \& Knight, 2009). In Canada, an estimated 3.2 million youth between the ages of 12-19 are at risk for developing depression, with $5 \%$ of male and $12 \%$ of female adolescents having experienced one major depressive episode (MHCC, 2013). Adolescence also represents a high-risk period for the first onset of suicidal behavior, as the rate of suicidal ideation (i.e., thinking about, considering, or planning suicide) jumps from less than $1 \%$ at age 10 to a staggering $17 \%$ by age 18 in the United States (Nock et al., 2013). Moreover, as many as 8\% of adolescents diagnosed with major depressive disorder have completed suicide by early adulthood (NIMH, 2019), making suicide the second leading cause of death in youth aged 15 to 24 in both the United States (Drapeau \& McIntosh, 2018) and Canada (Statistics Canada, 2018).

Additionally, Canadian youth aged 15 to 24 are more likely to experience mental illness and/or substance use disorders than any other age group (Pearson et al., 2013). For instance, problematic alcohol use (i.e., heavy drinking, or drinking that is accompanied by adverse consequences) for depressed adolescents has become increasingly prevalent and debilitating (Windle \& Davies, 1999). Although the causal mechanism underlying the relationship between adolescent depression and alcohol problems is not 
thoroughly understood, the extant research indicates that depression and alcohol problems act as risk factors for each other (see Swendsen \& Merikangas, 2000 for a review). For instance, longitudinal research demonstrated that depression in early adolescence is predictive of an increased risk of early alcohol use and alcohol-related problems (i.e., negative consequences associated with alcohol use such as impaired driving, school problems, and reckless sexual behaviors (White \& Labouvie, 1989)) in mid to late adolescence, with major depressive disorder doubling the odds of first-time alcohol use by age 14 (King et al., 2004). Additionally, comorbid depression and alcohol problems yield higher risk of suicide and greater social and personal impairment as well as other psychiatric conditions (Davis et al., 2008). Given the high rate of comorbidities between depression, alcohol problems, and suicidality in adolescence, it is imperative that researchers and clinicians develop a better understanding of the various risk and protective factors for these serious mental health challenges.

\section{The Role of Family Functioning in Adolescent Psychopathology}

A review of the extant literature suggests family functioning has an important role in the onset and trajectory of emotional and behavioral problems in adolescents, including depression, suicide behaviors, and alcohol use problems (Chan et al., 2013; Davies \& Windle, 1997; Sheeber et al., 1997). According to the family systems theory (Bowen, 1974), healthy family functioning or dysfunction is attributed to the ongoing complex interplays between family members, relationships and family system. These transactional patterns of the family system are important in shaping the behavior of individual family members (Bowen, 1974; Epstein et al., 1983). Through the lens of family systems theory, adolescent psychopathology can be interpreted as an indicator of impaired functioning within the larger family system as a whole (Bowen, 1974). For instance, one of the most distinctive risk factors associated with comorbid adolescent depression and alcohol problems is the perceived lack of family support (Chan et al., 2013; Davis et al., 2008; Sheeber et al., 1997; Windle \& Davies, 1999). Compared to adolescents with either depression or alcohol-related problems, adolescents with comorbid depression and alcohol-related problems reported the lowest levels of family social support (Windle \& Davies, 1999). Similarly, there is compelling evidence that family discord during adolescence significantly increases the risk of depression among adolescent girls, who are then more likely to turn to alcohol or other substances to alleviate depressive symptoms (Chan et al., 2013; Ohannessian et al., 2016). Prior research has also established prospective associations between adolescents' reports of family relationships (e.g., affective responsiveness, cohesion, and conflict) and subsequent adolescent alcohol and drug problems (Chan et al., 2013; McKay et al., 1991; Ohannessian et al., 2016).

Research also suggests that adolescent-reported family functioning in affective responsiveness is particularly relevant to adverse psychological consequences in adolescents, such as depression and alcohol use problems (e.g., McKay et al., 1991; Tamplin \& Goodyer, 2001). Familial affective responsiveness refers to the degree to which interactions between family members are characterized by warmth, affection, and emotional expressivity (Epstein et al., 1983). Affectively responsive parenting, whereby parents appropriately respond to children's expressions of feelings and needs, is critical in fostering a positive family emotional climate and setting up an early foundation for healthy development 
(Eisenberg et al., 2004). When children live in a consistent, responsive environment in which they feel nurtured and accepted, they feel emotionally secure and free to express their feelings because they are certain their emotional needs will be met. Indeed, children's perceptions of familial affective responsiveness are associated with greater use of active, support-seeking emotional regulatory strategies and fewer internalizing and externalizing problems (Eisenberg et al., 2004). In contrast, children whose parents minimize or discourage their emotion expression or punish them for expressing negative emotions are more emotionally reactive, more intense in emotional expression, and more likely to engage in avoidant emotion regulation strategies and escape tactics (e.g., problem drinking) to cope with distress (Eisenberg et al., 2004). For instance, adolescents from families low in affective responsiveness exhibit greater emotional distress and more drug- and alcohol-related problems (e.g., Chan et al., 2013; McKay et al., 1991; Tamplin \& Goodyer, 2001). Therefore, given the critical role of parental and familial responsiveness in adolescents' healthy social and emotional development, parent factors and characteristics that are closely related to familial affective responsiveness may be particularly relevant to a better understanding of adolescent psychological maladjustment.

\section{Parental Perfectionism and Adolescent Psychological Outcomes}

Over the past two decades, parental perfectionism and its associations with psychological wellbeing in children and adolescents have gained increasing empirical attention (e.g., Affrunti \& Woodruff-Borden, 2015; Mitchell et al., 2013; Soenens et al., 2005; 2006). During parent-child interaction tasks (e.g., solving difficult puzzles, preparing a speech), parents high in perfectionistic concerns (e.g., socially prescribed perfectionism, concern over mistakes, and doubts about actions) were more preoccupied with the negative consequences of the child's mistakes. In addition, these parents also displayed more controlling behaviors toward the child (e.g., giving explicit directives/commands, choice making, and behavior regulation), and used more negative emotion words during the tasks. Furthermore, the relationship between parental perfectionistic concerns and child anxiety was mediated by parents' controlling behaviors and/or their use of negative emotion words during the interaction tasks (Affrunti \& WoodruffBorden, 2015; Mitchell et al., 2013). In a study of high school students and their parents, Soenens et al. (2006) demonstrated that parental psychological control mediated the relationships between parental perfectionistic concerns and adolescent emotional wellbeing as assessed by measures of depression, loneliness, and low self-esteem.

In a recent meta-analytic review that included 14 studies on parents' trait perfectionism (i.e., perfectionistic strivings and perfectionistic concerns) and child/adolescent psychological outcomes, Lilley et al. (2020) found small but significant (positive) associations between parental perfectionistic concerns and child/adolescent psychological outcomes such as depression and anxiety, while controlling for parental perfectionistic strivings (e.g., self-oriented perfectionism). Perfectionistic concerns (e.g., socially prescribed perfectionism) also significantly increases the risk of parental burnout (Sorkkila \& Aunola, 2020). Additionally, the relationship between parental perfectionistic concerns and burnout is at least partly explained by parents' tendency to suppress or hide their emotions from their children (Lin \& Szczygiel, 2022). 
In sum, the extant research provides support for the notion that parents with elevated trait perfectionism engage in behaviors (e.g., psychological control, conditional approval) with the underlying message that the child must be perfect or appear perfect, and that mistakes and failures are distressing and intolerable (Affrunti \& Woodruff-Borden, 2015; Mitchell et al., 2013; Soenens et al., 2005; 2006). These authors concluded that parental perfectionistic concerns may be a core vulnerability factor for poor child/adolescent psychological outcomes (Lilley et al., 2020). Taken together, these findings highlight the role of trait perfectionism in parents as a potential vulnerability factor in child and adolescent psychopathology.

\section{Perfectionistic Self-Presentation and the Perfectionism Social Disconnection Model}

Despite the well-documented links between parental perfectionistic concerns (e.g., socially prescribed perfectionism) and adolescent psychological distress (see Lilley et al., 2020 for a review), no study has examined the role of parental perfectionistic self-presentation in adolescent psychological functioning. Perfectionistic self-presentation, as conceptualized by Hewitt and colleagues (2003), reflects the interpersonal expressions of perfectionism and consists of three distinct components: 1) perfectionistic self-promotion is driven by the need to appear perfect or to project an image of perfection to others, 2) nondisplay of imperfection is propelled by the need to avoid appearing as imperfect or flawed via avoidance of situations in which mistakes or flaws may be exposed or scrutinized, and 3) nondisclosure of imperfection is driven by the need to avoid verbally expressing or admitting to distress, mistakes, and any perceived imperfections due to fear of negative evaluation (Hewitt et al., 2003). The Perfectionism Social Disconnection Model (PSDM; Hewitt et al., 2017) offers a useful theoretical framework by suggesting that interpersonal components of perfectionism (e.g., perfectionistic self-presentation) leads to psychological problems by engendering interpersonal problems and social disconnection. Specifically, the PSDM asserted that individuals high in perfectionistic self-presentation engage in behaviors (e.g., hyper-criticalness, psychological control, and a lack of emotional sensitivity) that can give rise to problems with social or emotional connectedness (e.g., loneliness, social isolation, and interpersonal problems). This heightened sense of disconnectedness in turn increases the risk of negative psychological outcomes such as depression and suicide (Hewitt et al., 2017).

In support of the PSDM (Hewitt et al., 2017), perfectionistic self-presentation has been positively linked with indicators of social disconnection (e.g., loneliness, social anxiety, and interpersonal hopelessness), which in turn, predicted increased depressive symptoms and suicidal ideation in studies involving community adults (Rnic et al., 2021; Robinson et al., 2021) and adolescents (Goya Arce \& Polo, 2017). However, the extant research has focused exclusively on perfectionistic self-presentation in relation to the individual's own psychological or interpersonal functioning. To our knowledge, no study to date has tested the PSDM in the context of dyadic (e.g., parent-adolescent) or family relationships. Moreover, there is a lack of research on the role of family relationships in the context of parental perfectionism and adolescent psychopathology.

\section{The Current Study}


The general aim of this study was therefore to address these important gaps in the literature by exploring the associations between parental perfectionistic self-presentation, perceived family functioning in affective responsiveness, and adolescent psychological outcomes (i.e., depressive symptoms, suicide ideation, and alcohol-related problems) in a group of psychiatric adolescents and their parents. By examining family functioning in affective responsiveness, as a measure of social connectedness between family members, we aimed to shed more light on the mechanism by which parental perfectionistic self-presentation is associated with adolescent maladjustment. Notably, we included both adolescents' and parents' reports of familial affective responsiveness to provide multi-informant assessments of family functioning. This is crucial because of well-documented discrepancies between adolescent and parent reports about family relationships (see De Los Reyes et al., 2019 for a review). Additionally, parents high in perfectionistic self-presentation may underestimate or minimize the extent of family dysfunction due to their tendency to downplay or hide personal problems and distress (Hewitt et al., 2003). Assessing both adolescents' and parents' perceptions of family functioning can therefore add valuable insight into the dynamics of the parent-adolescent relationships.

Drawing on the PSDM (Hewitt et al., 2017) and prior research on parental perfectionism and adolescent psychopathology (Lilley et al., 2020), we hypothesized that parental perfectionistic self-presentation would be associated with 1) greater perceptions of family dysfunction in affective responsiveness, and 2) more severe adolescent psychological maladjustment as indicated by measures assessing depression, suicide ideation, and alcohol-related problems, and that 3 ) the positive associations between parental perfectionistic self-presentation and measures of adolescent psychopathology (i.e., depressive symptoms, suicide ideation, and alcohol-related problems) would be accounted for by greater perceived family dysfunction in affective responsiveness.

\section{Method}

\section{Participants}

\section{Adolescents}

The sample consisted of 58 adolescents ( 14 males and 41 females, and three adolescents did not disclose their gender). All participants were patients in the psychiatric units of a large children's hospital in Canada. Participants ranged in age from 13 to 19 years $(M=15.52, S D=1.41)$. Approximately $82.1 \%$ of the adolescents identified as Caucasian, $10.7 \%$ identified as Asian, and the remaining $7.1 \%$ identified as First Nations or Hispanic Canadians. All participants had the Diagnostic and Statistical Manual of Mental Disorders-IV-TR (DSM-IV-TR; APA, 1994) diagnoses of depressive disorders, including Major Depressive Disorder (64.3\%), Dysthymic Disorder (16.1\%), or Depressive Disorder NOS (19.6\%) as assessed by the Schedule for Affective Disorders and Schizophrenia for School-Age Children - Present and Lifetime Version (K-SADS-PL; Kaufman et al., 1996). Regarding comorbid diagnoses, $25.9 \%$ of the participants had a comorbid anxiety disorder, $7.4 \%$ had a comorbid behavioral disorder, $5.5 \%$ had a 
comorbid alcohol use disorder, and $1.8 \%$ had a comorbid eating disorder. Potential participants with current psychotic symptoms or developmental difficulties were excluded from the study.

\section{Parents}

A total of 58 parents (51 mothers and four fathers, and three parents did not identify their gender) participated alongside the adolescents. Parents ranged in age from 30 to 58 years $(M=46.08, S D=$ 5.74). With respect to marital status, $78.2 \%$ of the parents were married or cohabiting, $7.3 \%$ were single, $14.5 \%$ were divorced, separated, or widowed at the time of the study. Additionally, $20.8 \%$ of the parents finished high school or equivalent, $26.4 \%$ completed college or technical school, $35.8 \%$ acquired undergraduate degrees, and $15.7 \%$ received graduate degrees

\section{Measures}

\section{Adolescent Diagnostic Interview}

The Schedule for Affective Disorders and Schizophrenia for School-Age Children - Present and Lifetime Version (K-SADS-PL; Kaufman et al., 1996) is a semi-structure diagnostic interview designed to assess current and lifetime episodes of psychopathology in children and adolescents according to DSM-IV-TR criteria. Summary ratings are derived from the interviews with both the adolescent and a primary caregiver and all the relevant information available. The items are rated on a 4-point scale where a score of 0 indicates lack of adequate information and scores from 1 to 3 reflect the degree of symptom severity. Specifically, score 1 connotes lack of symptoms, a score 2 equates to subthreshold levels of symptomatology, and a score of 3 reflects symptoms meeting threshold criteria. If threshold criteria are met for any of the symptoms in the initial screening interview, additional information was gathered during a follow-up interview for an accurate diagnosis.

Diagnostic interviews were conducted by a psychology graduate student or a BA-level psychology student who had undergone extensive trainings in clinical interviewing and the K-SADS-PL administration. Satisfactory inter-rater reliability was established between the two interviewers for $20 \%$ of the interviews on symptom ratings (ICC $=.97)$, presence of diagnosis ( $100 \%$ agreement), and assigned diagnoses ( $86 \%$ agreement). Previous research demonstrated that combining parental and adolescent reports of psychopathology, should there be discrepancies, is an effective counter measure (Piacentini et al., 1992). As such, symptoms were considered present if either parent or adolescent endorsed any of the symptoms. In case of discrepant diagnoses arising from inconsistent reports from parent and adolescent, adolescents' psychiatrist's diagnosis was used.

\section{Adolescent Alcohol Problems}

Adolescents' alcohol-related problems were measured by the Rutgers Alcohol Problem Index (RAPl; White \& Labouvie, 1989). The RAPI is a 23-item measure assessing the occurrence of specific alcohol-related problems (e.g., "not able to do your homework or study for a test," "getting into fights," and "been in trouble with the police for having or drinking alcohol") in adolescents and young adults. Each item is 
rated from 0 (none) to 3 (more than 5 times). The RAPI encompasses items assessing a range of negative psychological, social, academic, and physical health consequences associated with alcohol use. The RAPI can be used over various timeframes while maintaining favorable psychometric properties, with test-retest reliabilities ranging from $r=.83$ (one month) to $r=.88$ (one year). In this study, adolescents were asked to indicate the number of times they have experienced each alcohol-related problem within the past year. Higher scores indicate greater occurrences of alcohol-related negative consequences.

\section{Adolescent Suicidal Ideation}

Adolescents' suicidal ideation was assessed by the Suicidal Ideation Questionnaire (SIQ; Reynolds, 1987). The SIQ consists of 30 items intended to assess thoughts about suicide in youth during the past month. Adolescents rated the frequency with which each thought occurred to them on a 7-point Likert type scale from 1 (never having the thought) to 7 (having the thought almost every day). Higher scores reflect greater suicide ideation. Internal consistency reliability is high $(a>0.95)$ and test-retest reliability is adequate $(r=0.72)$ over 4 weeks, a finding that is consistent with the state nature of the scale (Reynolds, 1987).

\section{Parental Perfectionistic Self-Presentation}

Parents' perfectionistic self-presentation was measured by the Perfectionistic Self-Presentation (PSPS; Hewitt et al., 2003). The PSPS is a 27-item scale assessing perfectionistic self-presentation styles in adults, and it consists of the following subscales: perfectionistic self-promotion (10 items, e.g., "I try always to present a picture of perfection"), nondisplay of imperfection (10 items, e.g., "I will do almost anything to cover up a mistake"), and nondisclosure of imperfection (7 items, e.g., "I never let others know how hard I work on things"). Parents responded to all items on a scale from 1 (disagree strongly) to 7 (agree strongly). The three subscales have shown to be internally consistent yet distinct from the trait perfectionism dimensions assessed by the Hewitt and Flett's Multidimensional Perfectionism Scale (Hewitt \& Flett, 1991).

\section{Parent and Adolescent Depressive Symptoms}

Symptoms of depression were assessed by the Beck Depression Inventory - $2^{\text {nd }}$ Edition (BDI-Il; Beck et al., 1996) for both parents and adolescents. The BDI-II is a 21 -item inventory that assesses severity of depression over the past two weeks in adults and youth aged 13 years and above. Symptoms are assessed with a 4-point rating scale for which a rating of zero indicates that a symptom is not present (e.g., "I do not feel sad") and a rating of three indicates the most severe form of a symptom (e.g., "I am so sad or unhappy that I can't stand it").

\section{Parent-and Adolescent-reported Familial Affective Responsiveness}

Parents and adolescents indicated their perceptions of family functioning in affective responsiveness using the McMaster Family Assessment Device (MFAD; Epstein et al., 1983). The MFAD is a self-report 
questionnaire designed to be completed independently by family members over the age of 12 years. The 60-item MFAD consists of six subscales assessing six dimensions of family functioning and a seventh subscale assessing general family functioning. In this study, we focused only on the subscale assessing family functioning in affective responsiveness. The 6-item affective responsiveness subscale evaluates the extent to which the family responds to a range of stimuli with the appropriate quality and quantity of feelings (e.g., "we are reluctant to show our affection for one another"). Participants rate each item on a 4-point scale (1 = strongly agree, 4 = strongly disagree), with higher scores indicating more problematic family functioning.

\section{Procedure}

Ethical approval for this study was obtained from the University of XX Behavioral Research Ethics Board and the Children's and Women's Research Ethics Board at the university-affiliated children's hospital serving one of Canada's largest metropolitan areas. All adolescent participants were currently undergoing psychiatric treatment at the Mood Disorders Clinic at the hospital. Adolescents between the ages of 13-19 and presenting with symptoms of a depressive disorder were identified via chart review and intake interview and invited to participate in the study. The nature of the study was explained as voluntary and confidential. It was also made clear to the participants that their involvement with the study was separate from families' original reasons for visiting the units, and that a decision not to participate would have no impact on their existing services. After obtaining informed parental consent and adolescent assent for the study, adolescents and their parents completed the diagnostic interview (i.e., K-SADS-PL) and self-report measures in separate sessions. Upon completion of the study, adolescents were provided with a small honorarium in appreciation for their time. Parents were reimbursed for transportation costs incurred during the study. All participants were debriefed at the end of the study.

\section{Analytic Plan}

Before conducting further analyses, we examined the data for missing values, univariate and multivariate outliers, the presence of curvilinear relationships as well as for the assumption of normality and collinearity (Tabachnick \& Fidell, 2007). Next, to investigate the first two hypotheses that parental perfectionistic self-presentation would be associated with 1) greater perceptions of family dysfunction in affective responsiveness and 2) poorer adolescent psychological adjustment as indicated by measures of depression, suicidal ideation, and alcohol-related problems, we examined zero-order correlation coefficients for the variables of interest.

Then, we tested our third hypothesis that the association between parental perfectionistic selfpresentation and adolescent psychological outcomes would be mediated by greater adolescent-reported family dysfunction in affective responsiveness. Because of the small sample size $(N=58)$, we tested three separate models with adolescent depressive symptoms, suicidal ideation, and alcohol-related problems as the dependent variable, respectively. We conducted path analyses with both latent and observed variables and examined indirect (i.e., mediated) effects of our path models. Additionally, parents' 
symptoms of depression were covaried in the path analyses to control for the effects of parental depression on adolescent outcomes.

We checked the Chi-square results for significance of the path models and computed parameter estimates using a maximum likelihood estimation method. An optimal model fit was evaluated using the following criteria: a root mean-square error of approximation (RMSEA) of 0.05 or less, an upper RMSEA's $90 \%$ confidence interval bound of 0.08 or less, a comparative fit index (CFI) and a Tucker-Lewis index (TLI) of 0.95 or more, and a standardized root mean squared residual (SRMR) of 0.05 or less (Hu \& Bentler, 1999). The magnitude of both path and zero-order correlation coefficients was interpreted according to Cohen's criteria (small $\geq .10$, medium $\geq .30$, and large $\geq .50$; Cohen, 1988). Additionally, we reported the completely standardized indirect effects to represent the strength of the indirect effect. All indirect effects were subjected to bootstrap analyses with 10,000 bootstrap samples and a $95 \%$ confidence interval $(\mathrm{Cl})$. A bias-corrected bootstrap 95\% confidence interval for the product of these paths that does not include zero provides evidence of a significant indirect effect (Byrne, 2016). All path analyses were conducted using Analysis of Moment Structures (AMOS 26.0; Arbuckle, 2019), while all other analyses were completed by using IBM Statistical Package for Social Sciences (SPSS) v. 26.0. Finally, all statistical tests were two-tailed, and a $p$ value $\leq .05$ was considered statistically significant.

\section{Results}

\section{Preliminary Analyses and Descriptive Statistics}

Means, standard deviations, and Cronbach's alpha for all study variables are presented in Table 1. All measures evidenced a good internal reliability, with all $a>.70$. Descriptive statistics of all study variables were within one standard deviation of the means reported in previous studies involving similar populations (e.g., Beck et al., 1996; Epstein et al., 1983; Hewitt et al., 2003; White \& Labouvie, 1989). Missing data was minimal across all measures (i.e., $<2.0 \%$ ). Little's missing completely at random (MCAR) test was non-significant (Chi-square $=45.06, \mathrm{DF}=57, p=.87$ ), indicating the data was missing completely at random. We used an expectation maximization algorithm in SPSS 26.0 to impute missing data. Regarding univariate outliers, we found an out-of-range value for parental depression, whose scores were brought into range (Tabachnick \& Fidell, 2007). No multivariate outliers were found, while the assumption of collinearity was met. Most study variables were approximately normally distributed, with appropriate skewness and kurtosis values, except for adolescent alcohol-related problems and parental depression.

A closer examination of the data revealed that approximately $40 \%$ (23 out of 58 ) of the adolescents did not endorse any negative consequences associated with drinking in the past year, which produced a positively skewed distribution. Of the remaining $60 \%$ (35 out of 58 ) who reported negative consequences associated with drinking, 22\% (13 out of 58) met the cut-off (i.e., 15 and above on the RAPI) for being "high-consequence" drinkers. Hence, a square root transformation was conducted on the RAPI and 
parental depression, thereby normalizing the skewed distributions. The transformed data were used in subsequent analyses.

Next, we conducted three independent samples $t$-tests to determine whether there were significant differences in key adolescent variables (i.e., adolescent depression, suicidal ideation, and family dysfunction in affective responsiveness) between adolescents who endorsed negative consequences attributable to drinking and those who did not endorse any negative consequence. Specifically, independent samples $t$-tests revealed that adolescents $(n=35)$ who endorsed negative consequences attributable to alcohol also reported greater degrees of family dysfunction in affective responsiveness, $t(1,56)=2.149, p=.036, d=0.58$, compared to adolescents $(n=23)$ who did not endorse any alcoholrelated problems in the past year. Moreover, adolescents who experienced alcohol-related negative consequences reported significantly greater depressive symptoms than their counterparts who endorsed no alcohol-related problems, $t(1,56)=2.910, p=.005, d=0.78$. Similarly, adolescents who experienced alcohol-related negative consequences reported significantly higher levels of suicidal ideation compared to those who did not endorse any alcohol-related problems, $t(1,56)=3.63, p=.001, d=0.99$. In addition, a paired samples $t$-test indicated that adolescents reported significantly greater family dysfunction in affective responsiveness than did their parents, $t(1,57)=4.70, p<.001, d=0.61$. And finally, there were no significant age or gender differences in any of the study variables.

\section{Bivariate Correlations}

Prior to further analyses, bivariate scatterplots were also examined for the presence of curvilinear relationships and none were evident. As shown in Table 1, parental nondisclosure of imperfection was positively associated with adolescents' perceptions of family dysfunction in affective responsiveness, but not with parents' perceptions of family dysfunction in affective responsiveness (Hypothesis 1 ). Contrary to our hypothesis, none of the parental perfectionistic self-presentation subscales showed significant associations with adolescent ratings of depressive symptoms and suicidal ideation. Parental nondisclosure of imperfection, however, was positively associated with adolescent alcohol-related problems, with small effects (Hypothesis 2). Additionally, adolescent-reported family dysfunction in affective responsiveness was positively associated with adolescent depressive symptoms, alcoholrelated problems and suicidal ideation, with small to medium effects. In contrast, parent-reported family dysfunction in affective responsiveness was not significantly associated with any other variable. Finally, all three parental perfectionistic self-presentation subscales were positively related to parental depression, with small to medium effects (see Table 1).

\section{Mediation Analyses}

Next, we conducted three separate path analyses to investigate the hypothesis that adolescent-reported family dysfunction in affective responsiveness would mediate the relationship between parental 
perfectionistic self-presentation and adolescent depressive symptoms, suicidal ideation, and alcoholrelated problems while controlling for the effects of parental depression (Hypothesis 3 ).

As shown in Figure 1, all three mediation models had a good fit to the data: alcohol-related problems: $\chi^{2}$ (6) $=2.759, p=.84 ; \mathrm{RMSEA}=.00,90 \% \mathrm{Cl}[.00, .10] ; \mathrm{CFI}=1$; TLI = 1; SRMR = .026 (Figure 1a); depressive symptoms: $\chi^{2}(6)=1.827, p=.94 ; \mathrm{RMSEA}=.00,90 \% \mathrm{Cl}[.00, .046]$; CFI $=1$; TLI = 1; SRMR = .022 (Figure $1 \mathrm{~b})$; and suicidal ideation: $\chi^{2}(6)=1.203, p=.98$; RMSEA $=.00,90 \% \mathrm{Cl}[.00, .00]$; CFI $=1 ; \mathrm{TLI}=1$; SRMR $=$ .018 (Figure 1c).

Consistent with our hypothesis, results showed that parental perfectionistic self-presentation had a significant, positive direct effect on adolescent-reported family dysfunction in affective responsiveness, $\beta$ $=.38 ; p=.018$, with medium effects. Further, adolescent-reported family dysfunction in affective responsiveness had a significant, positive direct effect on all three dependent variables (DVs) (alcoholrelated problems: $\beta=.30 ; p=.028$; depressive symptoms: $\beta=.33 ; p=.020$; suicidal ideation: $\beta=.46 ; p<$ .001), with small-to-medium effects (see Figure 1). Finally, direct effects from parental perfectionistic selfpresentation to each of the three DVs did not reach statistical significance (all $p s>.05$ ). Bias-corrected bootstrapped tests of mediation evidenced that parental perfectionistic self-presentation had a significant indirect effect on adolescent outcomes through adolescent-reported family dysfunction in affective responsiveness, with small effects (adolescent alcohol-related problems: standardized indirect effect $=.116, p=.048,95 \% \mathrm{Cl}$ [.001 to .307$]$; depressive symptoms: standardized indirect effect $=.124, p$ $=.017,95 \% \mathrm{Cl}[.016$ to .302$]$; and suicidal ideation: standardized indirect effect $=.176, p=.006,95 \% \mathrm{Cl}$ [.047 to .345]). In keeping with Simmons et al. (2011)'s recommendations for reducing false-positive findings in psychological research, we conducted the same analyses and found the significant indirect effects remained with or without controlling for parental depression.

Taken together, parental perfectionistic self-presentation accounted for approximately $11.5 \%$ of the variance in adolescent-reported family dysfunction in affective responsiveness. In addition, the path model explained about $15 \%$ of the variance in alcohol-related problems, $11.7 \%$ in depressive symptoms, and $19.3 \%$ in suicidal ideation for the adolescents. Finally, we conducted three additional path analyses on the associations between parental perfectionistic self-presentation and adolescent depressive symptoms, suicidal ideation, and alcohol-related problems, respectively, via parent-reported family dysfunction in affective responsiveness. Bias-corrected bootstrapped tests of mediation revealed no significant indirect effects in the links between parent' perfectionistic self-presentation and adolescent psychological outcomes via parent-reported family dysfunction in affective responsiveness, after controlling for parental depression (due to space limitations, these results are available upon request).

\section{Discussion}

The current study extended the existing literature on parental perfectionism and adolescent psychological outcomes by investigating the associations between parental perfectionistic self-presentation, perceptions of familial affective responsiveness, and psychological outcomes in a sample of depressed 
psychiatric adolescents and their primary caregivers/parents. To our knowledge, this cross-sectional study was the first to investigate the links between parental perfectionistic self-presentation and adolescent psychological outcomes, as well as the mediating role of family functioning in these links. Specifically, we obtained partial support for the study hypotheses by finding that 1) parental nondisclosure of imperfection was positively associated with adolescents' perceptions of family dysfunction in affective responsiveness, 2) parental nondisclosure of imperfection was also significantly associated with adolescent alcohol-related problems, and finally, 3) parental perfectionistic selfpresentation exerted a significant indirect effect on adolescent psychological outcomes (i.e., depressive symptoms, suicidal ideation, and alcohol-related problems) via adolescent-reported family dysfunction in affective responsiveness, while controlling for parental depression. Taken together, our study contributes to the growing body of research in support of the PSDM (Hewitt et al., 2017) and highlights parental perfectionistic self-presentation as a potential risk factor for adolescent psychological maladjustment.

Consistent with prior research linking perfectionistic self-presentation with a diverse range of interpersonal distress and maladjustment (e.g., Goya Arce et al., 2017; Hewitt et al., 2003; Rnic et al., 2021; Robinson et al., 2021), we found a significant association between parental nondisclosure of imperfection and adolescents' perceptions of family dysfunction in affective responsiveness. These findings are in line with the PSDM (Hewitt et al., 2017) and previous studies demonstrating the detrimental effect of perfectionistic self-presentation on one's interpersonal functioning. Specifically, past research indicates that individuals with elevated nondisclosure of imperfection tend to withdraw and disengage especially when under stress, thereby leading to further distress, social isolation, and relationship dysfunction (e.g., Goya Arce \& Polo, 2017; Rnic et al., 2021; Robinson et al., 2021). Our findings are also congruent with the notion that parents high in nondisclosure of imperfection may be too preoccupied with their own mistakes and flaws to be sufficiently attuned and responsive to their depressed child's emotional needs and distress. In keeping with parent-adolescent discrepancies in reports of family relationships (De Los Reyes et al., 2019), our study showed that adolescents reported lower levels of familial affective responsiveness than did their parents. Moreover, we found no significant association between parental nondisclosure of imperfection and their own ratings of family dysfunction in affective responsiveness. This non-significant association not only validates the importance of assessing family functioning from both adolescents' and parents' perspectives, but it also raises the possibility that parents who avoid disclosure of their flaws and mistakes may not have the awareness of or the willingness to acknowledge problems with the ways in which family members emotionally respond to one another.

Additionally, parental nondisclosure of imperfection also displayed a positive association with adolescent alcohol-related problems, which is in line with the extant literature emphasizing the role of parental perfectionistic concerns (e.g., socially prescribed perfectionism) in adolescent psychological distress (Lilley et al., 2020). Furthermore, our path analysis results highlight the mediating role of familial affective responsiveness in the links between parental perfectionistic self-presentation and adolescent psychological maladjustment in the forms of depression, suicidal ideation, and alcohol-related problems. According to the PSDM (Hewitt et al., 2017), perfectionistic self-presentation represents a set of defensive 
interpersonal behaviors aimed at securing approval and admiration (perfectionistic self-promotion) and preventing humiliations and rejections by others (nondisplay of imperfection and nondisclosure of imperfection). Paradoxically, these very behaviors that aim to enhance one's social approval and standing and prevent rejections and alienation often promote feelings of shame, emotional detachment, and subjective and/or objective forms of disconnectedness from one's social environment (e.g., Goya Arce et al., 2017; Lin \& Szczygiel, 2022; Rnic et al., 2021; Robinson et al., 2021). By assessing familial affective responsiveness, as a measure of perceived emotional connectedness between family members, our study sheds more light on the mechanism by which parental perfectionism contributes to adolescent psychological maladjustment.

From a family systems perspective (Bowen, 1974; Epstein et al., 1983), parents who eschew disclosures of imperfections and distress can create or contribute to a family environment deprived of opportunities for emotional connectedness and intimacy. By not sharing or admitting to personal distress or perceived imperfections, parents with elevated perfectionistic self-presentation may inadvertently communicate to their adolescents that sharing one's flaws and distress is unacceptable, and such disclosures may bring embarrassment or even humiliation to oneself and one's own family. Parental perfectionistic selfpresentation may also reinforce adolescents' perceptions of the family members as lacking in emotional sensitivity and responsiveness with one another. Consequently, rather than turning to their parents for emotional support and comfort, adolescents struggling with depression may resort to excessive drinking and other maladaptive behaviors to cope with emotional pain and distress (e.g., Chan et al., 2013; King et al., 2004). Alternatively, considering the positive associations between parental perfectionistic selfpresentation dimensions and depressive symptoms, parents with elevated perfectionistic selfpresentation may be prone to parenting stress or burnout, which can also interfere with their ability to detect and respond to their adolescents' signals of distress (Gondoli \& Silverberg, 1997). Nevertheless, our study demonstrates that the significant indirect effects of familial affective responsiveness on parental perfectionistic self-presentation and adolescent psychological maladjustment remained even after controlling for parental depression.

\section{Clinical Implications}

From a clinical standpoint, our study highlights the importance of considering parental perfectionism and family functioning when evaluating and treating clinically depressed adolescents. For parents with elevated perfectionistic self-presentation, having an adolescent with a diagnosis of clinical depression may be viewed as a profound disappointment or failure for themselves and their families. Dang et al. (2020) found that all three perfectionistic self-presentation subscales displayed significant associations with greater perceived mental health stigma and a lower willingness to seek professional help. Therefore, high levels of parental perfectionistic self-presentation can present challenges for adolescents and their families in terms of acknowledging the severity of mental health and family problems and accessing mental health services when needed. When treating adolescent depression and other comorbidities (e.g., suicidality, alcohol use problems), there needs to be more emphasis on evaluating and improving parents' 
capacity to recognize and appropriately respond to their depressed adolescents' emotional needs and distress.

\section{Limitations And Future Directions}

There are a few limitations that warrant further investigations. First, the study is cross-sectional and hence no causal conclusions can be drawn. Future longitudinal studies or causal designs are needed to ascertain the causal relationships between parental perfectionistic self-presentation, familial affective responsiveness, and adolescent psychological outcomes. Second, our sample size $(n=58)$ may be too small to detect some of the hypothesized associations between parental perfectionistic self-presentation and adolescent depressive symptoms and suicidal ideation. As well, it remains unclear how representative our adolescent sample is of the general psychiatric adolescent population since $100 \%$ of our participants were diagnosed with a DSM-IV depressive disorder. On a related note, our sample consists of predominantly Caucasian adolescent girls and their mothers, thereby precluding investigations of important gender- and culture-specific risk and protective factors associated with problem drinking in depressed adolescents.

Future research should also include measures of adolescent and parent anxiety and other comorbid disorders with adolescent depressive disorders, as well as a more complete assessment of problem drinking in adolescents (e.g., alcohol consumption, binge drinking, and motivations for alcohol use). Furthermore, given the differential effects of maternal and paternal behaviors on male versus female adolescents (Davies \& Windle, 1997), a more comprehensive understanding of the effects of parental perfectionistic self-presentation on familial affective responsiveness and adolescent psychological functioning may require knowing the gender of the parent-adolescent dyads. Additionally, observational studies are necessary to better understand the behavioral and emotional displays of parental perfectionistic self-presentation (e.g., hostile-dominant behaviors, a lack of emotional responsiveness) during parent-adolescent and/or family interactions. Finally, in efforts to develop more targeted treatment and prevention interventions, future studies should explore different pathways linking parental perfectionism to adolescent psychopathology, which will mostly likely involve a dynamic interplay among specific adolescent, parent, and familial factors.

\section{Conclusions}

Despite these limitations, our study demonstrates that, in line with the theory (PSDM; Hewitt et al., 2017) and the extant literature on the role of parental perfectionism in child and adolescent psychopathology (Lilley et al., 2020), parental perfectionistic self-presentation may represent a potential risk factor for problematic family functioning in affective responsiveness, which in turn is related to adolescent psychological distress. Specifically, our findings suggest that adolescent but not parent-reported family dysfunction in affective responsiveness mediated the relations between parental perfectionistic selfpresentation and adolescent depressive symptoms, suicidal ideation, and alcohol-related problems while controlling for parental depression. As such, understanding parental perfectionism and family 
relationships may represent important targets for family-based interventions for adolescent depression and other comorbid conditions.

\section{Declarations}

\section{Author Note}

We have no known conflict of interest to disclose.

This research was supported by grants from the Social Sciences and Humanities Research Council of Canada (SSHRC; 410-2009-1050; 435-2015-0412) awarded to the third author.

Correspondence concerning this article should be addressed to Paul L. Hewitt, Department of Psychology, University of British Columbia, 2136 West Mall, Vancouver, British Columbia, Canada, V6T 1Z4. Email: phewitt@pspych.ubc.ca

\section{References}

1. Affrunti, N. W., \& Woodruff-Borden, J. (2015). Parental perfectionism and overcontrol: Examining mechanisms in the development of child anxiety. Journal of Abnormal Child Psychology, 43, 517529. https://doi.org/10.1007/s10802-014-9914-5

2. American Psychiatric Association [APA]. (1994). Diagnostic and statistical manual of mental disorders ( $4^{\text {th }}$ ed.). American Psychiatric Association.

3. Arbuckle, J. L. (2019). Amos (Version 26.0) [Computer software]. IBM SPSS.

4. Beck A. T., Steer, R. A., \& Brown, G. K. (1996). Manual for the Beck Depression Inventory-II. Psychological Corporation.

5. Bowen, M. (1974). Alcoholism as viewed through family systems theory and family psychotherapy. Annals of the New York Academy of Sciences, 233, 115-122. https://doi.org/10.1111/j.17496632.1974.tb40288.x

6. Byrne, B. M. (2016). Structural equation modeling with AMOS: Basic concepts, applications, and programming (3rd ed.). Routledge. https://doi.org/10.4324/9781315757421

7. Chan, G. C., Kelly, A. B., \& Toumbourou, J. W. (2013). Accounting for the association of family conflict and heavy alcohol use among adolescent girls: The role of depressed mood. Journal of Studies on Alcohol and Drugs, 74(3), 396-405. https://doi.org/10.15288/jsad.2013.74.396

8. Cohen, J. (1988). Statistical power analysis for the behavioral sciences ( $2^{\text {nd }}$ ed.). Hillsdale, NJ: Erlbaum.

9. Dang, S. S., Quesnel, D. A., Hewitt, P. L., Flett, G. L., \& Deng, X. (2020). Perfectionistic traits and selfpresentation are associated with negative attitudes and concerns about seeking professional psychological help. Clinical Psychology and Psychotherapy, 27(5), 621-629. https://doi.org/10.1002/cpp.2450 
10. Davies, P. T., \& Windle, M. (1997). Gender-specific pathways between maternal depressive symptoms, family discord, and adolescent adjustment. Developmental Psychology, 33(4), 657-668. https://doi.org/10.1037/0012-1649.33.4.657

11. Davis, L., Uezato, A., Newell, J. M., \& Frazier, E. (2008). Major depression and comorbid substance use disorders. Current Opinion in Psychiatry, 21(1), 14-

18. http://dx.doi.org/10.1097/YCO.0b013e3282f32408

12. De Los Reyes, A., Ohannessian, C. M., \& Racz, S. J. (2019). Discrepancies between adolescent and parent reports about family relationships. Child Development Perspectives, 13(1), 53-58. https://doi.org/10.1111/cdep.12306

13. Drapeau, C. W., \& McIntosh, J. L. (2018). U.S.A. suicide 2017: Official final data [Data set]. American Association of Suicidology. http://www.suicidology.org

14. Eisenberg, N., Smith, C. L., Sadovsky, A., \& Spinrad, T. L. (2004). Effortful control: Relations with emotion regulation, adjustment, and socialization in childhood. In R. F. Baumeister \& K. D. Vols (Eds.), Handbook of self-regulation: Research, theory, and applications (pp. 259-282). Guilford Press.

15. Epstein, N. B., Baldwin, L. M., \& Bishop, D. S. (1983). The McMaster family assessment device. Journal of Marital and Family Therapy, 9(2), 171-180. https://doi.org/10.1111/j.17520606.1983.tb01497.x

16. Gondoli, D. M., \& Silverberg, S. B. (1997). Maternal emotional distress and diminished responsiveness: The mediating role of parenting efficacy and parental perspective taking. Developmental Psychology, 33(5), 861-868. https://doi.org/10.1037/0012-1649.33.5.861

17. Goya Arce, A. B., \& Polo, A. J. (2017). A test of the Perfectionism Social Disconnection Model among ethnic minority youth. Journal of Abnormal Child Psychology, 45, 1181-1193. https://doi.org/10.1007/s10802-016-0240-y

18. Hewitt, P. L., \& Flett, G. L. (1991). Perfectionism in the self and social contexts: Conceptualization, assessment, and association with psychopathology. Journal of Personality and Social Psychology, 60(3), 456-470. https://doi.org/10.1037/0022-3514.60.3.456

19. Hewitt, P. L., Flett, G. L., Sherry, S. B., Habke, M., Parkin, M., Lam, R. W., McMurtry, B., Ediger, E., Fairlie, P., \& Stein, M. B. (2003). The interpersonal expression of perfectionism: perfectionistic selfpresentation and psychological distress. Journal of Personality and Social Psychology, 84(6), 13031325. https://doi.org/10.1037/0022-3514.84.6.1303

20. Hewitt, P. L., Flett, G. L., \& Mikail, S. F. (2017). Perfectionism: A relational approach to conceptualization, assessment and treatment. Guilford Press.

21. Hu, L.-t., \& Bentler, P. M. (1999). Cutoff criteria for fit indexes in covariance structure analysis: Conventional criteria versus new alternatives. Structural Equation Modeling, 6(1), 1-55. https://doi.org/10.1080/10705519909540118

22. Kaufman, J., Birmaher, B., Brent, D., Rao, U., Flynn, C., Moreci, P., Williamson, D., \& Ryan, N. (1997). Schedule for affective disorders and schizophrenia for school-age children - present and lifetime 
version (K-SADS-PL): Initial reliability and validity data. Journal of the American Academy of Child \& Adolescent Psychiatry, 36(7), 980-988. https://doi.org/10.1097/00004583-199707000-00021

23. Kaufman, J., Birmaher, B., Brent, D., Rao, U., \& Ryan, N. (1996). Schedule for Affective Disorders and Schizophrenia for School-Age Children - Present and Lifetime Version: Manual. University of Pittsburgh School of Medicine, Pittsburgh, PA.

24. King, S. M., lacono, W. G., \& McGue, M. (2004). Childhood externalizing and internalizing psychopathology in the prediction of early substance use. Addiction, 99(12), 1548-1559. https://doi.org/10.1111/j.1360-0443.2004.00893.x

25. Lilley, C., Sirois, F., \& Rowse, G. (2020). A meta-analysis of parental multidimensional perfectionism and child psychological outcomes. Personality and Individual Differences, 162, 110015. https://doi.org/10.1016/j.paid.2020.110015

26. Lin, G.-X., \& Szczygiel, D. (2022). Perfectionistic parents are burnt out by hiding emotions from their children, but this effect is attenuated by emotional intelligence. Personality and Individual Differences, 184, 111187. https://doi.org/10.1016/j.paid.2021.111187

27. McKay, J. R., Murphy, R. T., Rivinus, T. R., \& Maisto, S. A. (1991). Family dysfunction and alcohol and drug use in adolescent psychiatric inpatients. Journal of the American Academy of Child \& Adolescent Psychiatry, 30(6), 967-972 https://doi.org/10.1097/00004583-199111000-00015

28. Mental Health Commission of Canada [MHCC]. (2013). Making the case for investing in mental health in Canada. https://www.mentalhealthcommission.ca/English/media/3179

29. Merikangas, K. R., \& Knight, E. (2009). The epidemiology of depression in adolescents. In S. NolenHoeksema \& L. M. Hilt (Eds.), Handbook of depression in adolescents (pp. 53-73). Routledge.

30. Mitchell, J. H., Broeren, S., Newall, C., \& Hudson, J. L. (2013). An experimental manipulation of maternal perfectionistic anxious rearing behaviors with anxious and non-anxious children. Journal of Experimental Child Psychology, 116(1), 1-18. https://doi.org/10.1016/j.jecp.2012.12.006

31. National Institute of Mental Health [NIMH]. (2019, February). Major depression. https://www.nimh.nih.gov/health/statistics/major-depression.shtml

32. Nock, M. K., Green, J. G., Hwang, I., McLaughlin, K. A., Sampson, N. A., Zaslavsky, A. M., \& Kessler, R. C. (2013). Prevalence, correlates, and treatment of lifetime suicidal behavior among adolescents: Results from the National Comorbidity Survey Replication Adolescent Supplement. JAMA Psychiatry, 70(3), 300-310. http://dx.doi.org/10.1001/2013.jamapsychiatry.55

33. Ohannessian, C. M., Flannery, K. M., Simpson, E., \& Russell, B. S. (2016). Family functioning and adolescent alcohol use: A moderated mediation analysis. Journal of Adolescence, 49, 19-27. https://doi.org/10.1016/j.adolescence.2016.02.009

34. Patel, V., Flisher, A. J., Hetrick, S., \& McGorry, P. (2007). Mental health of young people: A global public health challenge. Lancet, 369(9569), 1302-1313. https://doi.org/10.1016/S0140-6736(07)60368-7

35. Pearson, C., Janz, T., \& Ali, J. (2013). Health at a glance: Mental and substance use disorders in Canada. Statistics Canada Catalogue no. 82-624-X. https://www150.statcan.gc.ca/n1/pub/82-624x/2013001/article/11855-eng.htm 
36. Piacentini, J. C., Cohen, P., \& Cohen, J. (1992). Combining discrepant diagnostic information from multiple sources: Are complex algorithms better than simple ones? Journal of Abnormal Child Psychology, 20, 51-63. https://doi.org/10.1007/BF00927116

37. Reynolds, W. M. (1987). Suicidal Ideation Questionnaire. Psychological Assessment Resources.

38. Rnic, K., Hewitt, P. L., Chen, C., Flett, G. L., Jopling, E., \& LeMoult, J. (2021). Examining the link between multidimensional perfectionism and depression: A longitudinal study of the intervening effects of social disconnection. Journal of Social and Clinical Psychology, 40(4), 277-303. https://doi.org/10.1521/jscp.2021.40.4.277

39. Robinson, A., Moscardini, E., Tucker R., \& Calamia, M. (2021). Perfectionistic self-presentation, socially prescribed perfectionism, self-oriented perfectionism, interpersonal hopelessness, and suicidal ideation in U.S. adults: Re-examining the Social Disconnection Model. Archives of Suicide Research. Advance online publication. https://doi.org/10.1080/13811118.2021.1922108

40. Sheeber, L., Hops, H., Alpert, A., Davis, B., \& Andrews, J. (1997). Family support and conflict: Prospective relations to adolescent depression. Journal of Abnormal Child Psychology, 25(4), 333344. https://doi.org/10.1023/A:1025768504415

41. Simmons, J. P., Nelson, L. D., \& Simonsohn, U. (2011). False-positive psychology: Undisclosed flexibility in data collection and analysis allows presenting anything as significant. Psychological Science, 22(11), 1359-1366. https://doi.org/10.1177/0956797611417632

42. Soenens, B., Elliot, A. J., Goossens, L., Vansteenkiste, M., Luyten, P., \& Duriez, B. (2005). The intergenerational transmission of perfectionism: Parents' psychological control as intervening variable. Journal of Family Psychology, 19(3), 358-366. https://doi.org/10.1037/0893-3200.19.3.358

43. Soenens, B., Vansteenkiste, M., Duriez, B., \& Goossens, L. (2006). In search of the sources of psychologically controlling parenting: The role of parental separation anxiety and parental maladaptive perfectionism. Journal of Research of Adolescence, 16(4), 539-559. https://doi.org/10.1111/j.1532-7795.2006.00507.x

44. Sorkkila, M., \& Aunola, K. (2020). Risk factors for parental burnout among Finnish parents: The role of socially prescribed perfectionism. Journal of Child and Family Studies, 29(3), 648-659. https://doi.org/10.1007/s10826-019-01607-1

45. Spear, L. P. (2014). Adolescents and alcohol: Acute sensitivities, enhanced intake, and later consequences. Neurotoxicology and Teratology, 41, 51-59. https://doi.org/10.1016/j.ntt.2013.11.006

46. Statistics Canada. (2018). Deaths and age-specific mortality rates, by selected grouped causes [Data set]. https://www150.statcan.gc.ca/t1/tbl1/en/tv.action\%3fpid\%3d1310039201

47. Swendsen, J. D., \& Merikangas, K. R. (2000). The comorbidity of depression and substance use disorders. Clinical Psychology Review, 20(2), 173-189. https://doi.org/10.1016/S02727358(99)00026-4

48. Tabachnick, B. G., \& Fidell, L. S. (2007). Using multivariate statistics (5th ed.). Allyn \& Bacon/Pearson Education. 
49. Tamplin, A., \& Goodyer, I. M. (2001). Family functioning in adolescents at high and low risk for major depressive disorder. European Child \& Adolescent Psychiatry, 10(3), 170-

179. https://doi.org/10.1007/s007870170023

50. White, H. R., \& Labouvie, E. W. (1989). Towards the assessment of adolescent problem drinking. Journal of Studies on Alcohol and Drugs, 50(1), 30-37. https://doi.org/10.15288/jsa.1989.50.30

51. Windle, M., \& Davies, P. T. (1999). Depression and heavy alcohol use among adolescents: Concurrent and prospective relations. Development and Psychopathology,11(4), 823-844. https://doi.org/10.1017/S0954579499002345

\section{Tables}

Table 1 Descriptive Statistics and Bivariate Correlations for Adolescent and Parent Variables 


\begin{tabular}{|c|c|c|c|c|c|c|c|c|c|}
\hline & 1 & 2 & 3 & 4 & 5 & 6 & 7 & 8 & 9 \\
\hline $\begin{array}{l}\text { 1. Perfectionistic } \\
\text { Self-Promotion } \\
\text { (P) }\end{array}$ & - & $.66^{* *}$ & $.78^{\star *}$ & -.14 & $.40^{\star \star}$ & .13 & .00 & .20 & -.01 \\
\hline $\begin{array}{l}\text { 1. Nondisplay of } \\
\text { Imperfection }(P)\end{array}$ & & - & $.68^{\star *}$ & -.06 & $.39^{\star \star}$ & .08 & .01 & .20 & -.10 \\
\hline $\begin{array}{l}\text { 1. Nondisclosure of } \\
\text { Imperfection }(P)\end{array}$ & & & - & -.06 & $.36^{\star *}$ & $.27^{\star}$ & .04 & $.29^{*}$ & -.17 \\
\hline $\begin{array}{l}\text { 1. Depressive } \\
\text { Symptoms (A) }\end{array}$ & & & & - & -.18 & $.41^{\star *}$ & $.76^{\star *}$ & $.28^{\star}$ & .02 \\
\hline $\begin{array}{l}\text { 1. Depressive } \\
\text { Symptoms (P) }\end{array}$ & & & & & - & .17 & -.04 & -.04 & -.08 \\
\hline $\begin{array}{l}\text { 1. Alcohol-Related } \\
\text { Problem (A) }\end{array}$ & & & & & & - & $.42^{\star \star}$ & $.39^{* \star}$ & .02 \\
\hline $\begin{array}{l}\text { 1. Suicidal Ideation } \\
\text { (A) }\end{array}$ & & & & & & & - & $.43^{* \star}$ & -.02 \\
\hline $\begin{array}{l}\text { 1. Familial Affective } \\
\text { Responsiveness } \\
\text { (A) }\end{array}$ & & & & & & & & - & -.07 \\
\hline $\begin{array}{l}\text { 1. Familial Affective } \\
\text { Responsiveness } \\
\text { (P) }\end{array}$ & & & & & & & & & - \\
\hline$M$ & 34.55 & 38.08 & 20.00 & 30.58 & 10.09 & 8.69 & 62.09 & 2.46 & 1.96 \\
\hline$S D$ & 10.11 & 11.09 & 7.41 & 12.43 & 8.18 & 12.49 & 46.96 & 0.65 & 0.54 \\
\hline Range & $12-62$ & $16-63$ & $7-43$ & $4-55$ & $0-47$ & $0-52$ & $0-165$ & $\begin{array}{l}1- \\
3.83\end{array}$ & $1-3$ \\
\hline$a$ & .82 & .80 & .75 & .92 & .91 & .81 & .98 & .84 & .85 \\
\hline
\end{tabular}

Note. ${ }^{*} p<.05,{ }^{\star *} p<.01 . N=58$ parent-adolescent dyads. (A) denotes adolescents; $(\mathrm{P})$ denotes parents. Familial Affective Responsiveness = McMaster Family Assessment Device (MFAD) affective responsiveness subscale. Higher scores on Familial Affective Responsiveness indicate more problematic family functioning. 

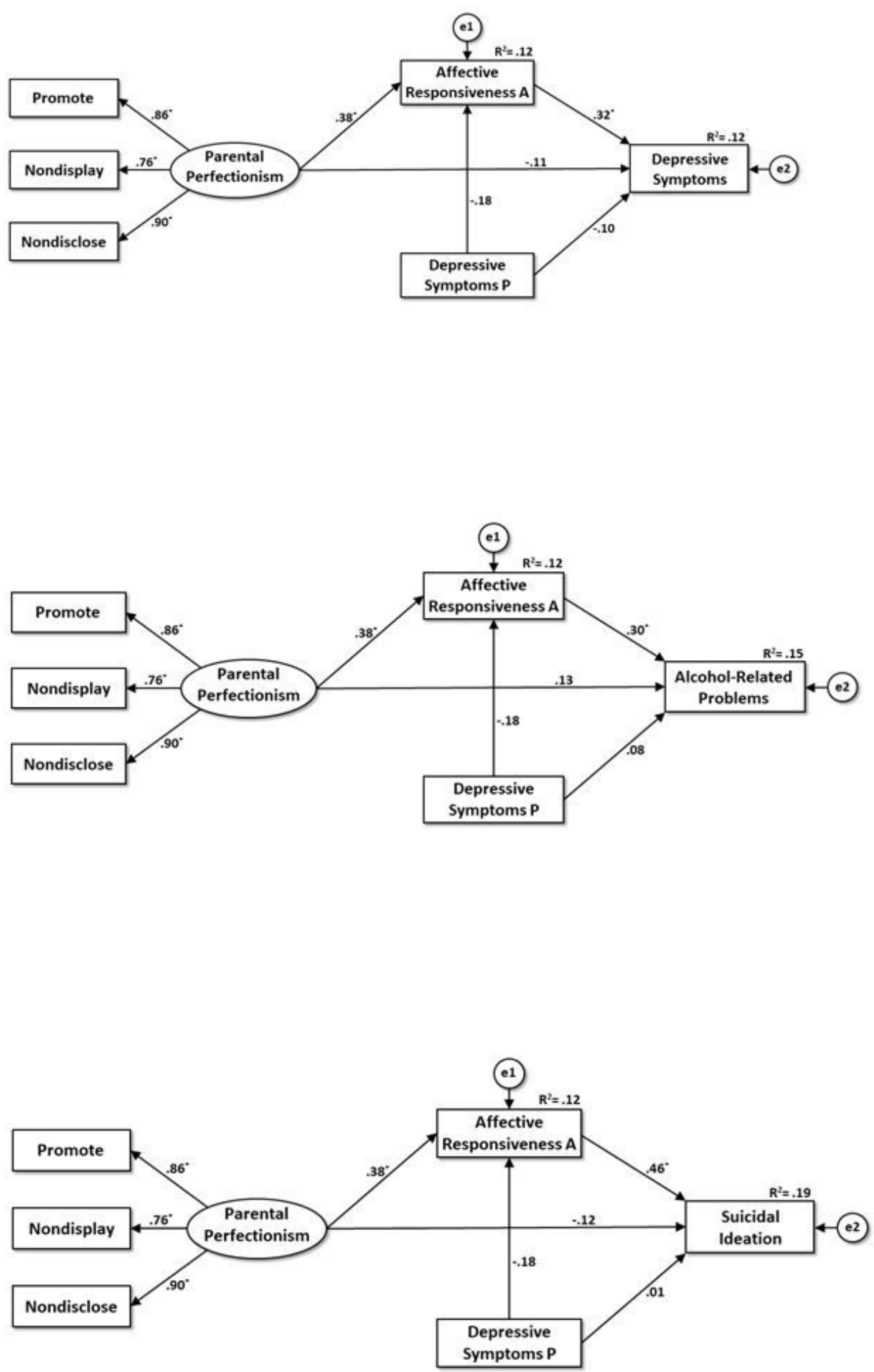

\section{Figure 1}

Parental perfectionistic self-presentation exerts an indirect effect on adolescent alcohol-related problems (Figure 1a), depressive symptoms (Figure 1b), and suicidal ideation (Figure 1c) via adolescent-reported family dysfunction in affective responsiveness, while controlling for parental depression. 
Note. ${ }^{*} p<.05$. (A) denotes adolescents; $(\mathrm{P})$ denotes parents. Promote $=$ perfectionistic self-promotion; nondisplay $=$ nondisplay of imperfection; nondisclosure $=$ nondisclosure of imperfection. Proportion of variance squared $\left(R^{2}\right)$ is presented within each variable. The covariance among parental perfectionistic self-presentation and parental depression is modeled but not shown for ease of interpretation. 